\title{
Stickler Syndrome (SS): Laser Prophylaxis for Retinal Detachment (Modified Ora Secunda Cerclage, OSC/SS)
}

This article was published in the following Dove Press journal: Clinical Ophthalmology

\begin{abstract}
Robert E Morris, (iD) $1-4$
Edward Scott Parma, (iD ${ }^{5}$

Nathaniel H Robin, ${ }^{6}$ Mathew R Sapp, ${ }^{1-4}$

Matthew H Oltmanns, (iD) ${ }^{1-4}$

Matthew R West, ${ }^{1-4}$ Donald C Fletcher, ${ }^{7,8}$

Ronald A Schuchard, ${ }^{9}$ Ferenc Kuhn $2,10,11$

'Retina Specialists of Alabama, Birmingham, AL, USA; ${ }^{2}$ Helen Keller Foundation for Research and Education, Birmingham, AL, USA;

${ }^{3}$ University of Alabama at Birmingham (UAB), Department of Ophthalmology, Birmingham,

AL, USA; ${ }^{4} U A B$ Callahan Eye Hospital, Birmingham, AL, USA; ${ }^{5}$ Retina Specialists of Alabama Montgomery, Montgomery, AL, USA; ${ }^{6} U A B$ Department of Genetics, Birmingham, AL, USA; ${ }^{7}$ University of Kansas Medical Center, Department of Ophthalmology and KU Eye Center, Kansas City, KS, USA; ${ }^{8}$ Retina Consultants of Southwest Florida, Ft. Myers, FL, USA; ' Envision Research Institute, Wichita, KS, USA; ${ }^{10}$ Milos Eye Hospital, Belgrade, Serbia;

"University of Pécs, Department of

Ophthalmology, Pécs, Hungary
\end{abstract}

\section{Video abstract}

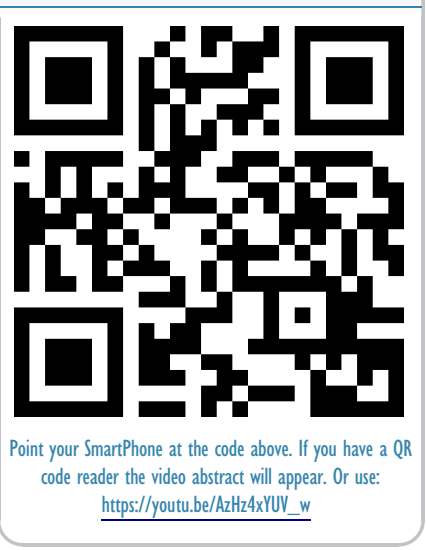

Correspondence: Robert E Morris Retina Specialists of Alabama, 2208 University Blvd, Birmingham, AL 35233. USA

Tel +I (205) 558-2598

Fax + I (205) 558-2596

Email rmorris@retinanetwork.com
Purpose: To introduce a novel technique of encircling laser prophylaxis (ora secunda cerclage Stickler syndrome, OSC/SS) to prevent rhegmatogenous retinal detachment (RRD) in Stickler syndrome eyes.

Patients and Methods: After first eye RRD at age 50 and at age 18, respectively, a 53-yearold father and his 22-year-old son with type 2 SS (STL2) gave informed consent and underwent OSC/SS prophylaxis, performed in each fellow eye. A 26-year-old STL2 daughter then suffered first eye retinal detachment and similarly chose fellow eye OSC/SS prophylaxis. A second son, 28 years of age with STL2, chose OSC/SS prophylaxis in both eyes.

Results: The three OSC/SS treated fellow eyes have gone 12 years, 11 years, and 8 years without RRD. STL1 and less common STL2 eyes are known to have a similar rate of RRD, and $80 \%$ of STL1 fellow eyes develop RRD at a median of 4 years in the absence of prophylaxis. Moreover, five of six (83\%) known STL2 family members suffered RRD, only the STL2 son with bilateral OSC/SS remaining bilaterally attached. All five OSC/SS treated eyes (average 8.7 years post-prophylaxis) retained preoperative visual acuity of 20/20 to 20/ 30 , with an average, asymptomatic reduction of meridional field in each eye to 50 degrees. In contrast, in the three eyes having suffered RRD prior to presentation, visual acuity ranged from $20 / 125$ to $8 / 200$ and average meridional field was 29 degrees.

Conclusion: Encircling grid laser (OSC) modified in Stickler eyes to encompass the ora serrata and extend posteriorly to and between the vortex vein ampullae (OSC/SS) is a reasonable RRD prophylaxis option to offer STL1 and STL2 patients as an alternative to no treatment or less effective prophylaxis. Because of rarity and severity, the ultimate proof of safety and efficacy will likely come not from randomized trials, but from a nonrandomized, prospective, cohort comparison study of such individual efforts.

Keywords: Stickler syndrome, SS, STL1, STL2, retinal detachment prevention, giant retinal tear, encircling laser prophylaxis, Ora Secunda Cerclage, OSC, OSC/SS

\section{Introduction}

Stickler syndrome (SS) is a heterogeneous inherited disorder of collagen formation with mutations primarily in the genes coding for type II collagen (type $1 \mathrm{SS}, \mathrm{STL} 1,80 \%$ of cases) and type XI collagen (type 2 SS, STL2, $<20 \%$ of cases). ${ }^{1,2}$ It is usually inherited in an autosomal dominant fashion and affects the eye, ear, and skeleton. A description of the important genetic aspects of Stickler syndrome was recently provided by Robin et al. ${ }^{1}$

Ocular-only or predominately ocular versions of SS have been increasingly recognized, emphasizing the importance of discovery by an ophthalmologist. ${ }^{3,4}$ 
Ocular findings in the STL1 and STL2 phenotype include early-onset myopia, a mostly liquid vitreous cavity from birth, ${ }^{5}$ membranous (STL1) or beading (STL2) vitreous opacities, ${ }^{3}$ radial perivascular retinal degeneration, ${ }^{4}$ foveal hypoplasia, ${ }^{6}$ early-onset cortical cataracts, ${ }^{7,8}$ and most importantly, retinal detachment that commonly occurs in the first three decades of life, even in infancy. ${ }^{9}$

Stickler syndrome is the leading cause of inherited rhegmatogenous retinal detachment (RRD). In Stickler patients, $\mathrm{RRD}$ is usually bilateral and is often caused by a giant retinal tear (GRT) at the ora serrata. ${ }^{9}$ Most Stickler detachments require multiple operations to repair, often with legally blind visual results despite final reattachment. ${ }^{10}$ It is thus an especially devastating disease for those affected.

In a series of 194 untreated STL1 patients with a mean age of 31.3 years retrospectively reported in 2014, the Cambridge (England) group observed an RRD prevalence rate of $53.6 \%$ (10.3\% unilateral and $43.3 \%$ bilateral), with a median time to first eye detachment of 18 years. In patients who had already suffered RRD in one eye before presenting, there was an $80 \%$ chance of a second eye detachment, at a median of 4 years after the first eye detachment. ${ }^{9}$

Against this natural course, Cambridge employed a single row of encircling cryopexy crossing the ora serrata for prophylaxis of GRT. Bilateral controls (no RRD) had a 5.0-fold increased risk of an $\mathrm{RRD}(\mathrm{p}<0.001)$ relative to bilateral-treated eyes matched for age and follow-up (mean 5.9 years). ${ }^{9}$ Prophylaxis failures $(9 \%)$ occurred at an average of 5.6 years after treatment, and the mean age at treatment was 21.5 years in failed cases. No long-term complications of encircling cryopexy were observed.

The (continuing) Cambridge prophylaxis experience of over four decades exceeds that of all other literature reports combined. As the only entity designated as a national center (United Kingdom) for this rare disease, its Stickler population is diverse and its prevalence figures are likely higher quality data than cross-study reviews of much smaller reports having inconsistent diagnostic and inclusion criteria. ${ }^{11}$ For example, $87.5 \%$ of clinically diagnosed Cambridge STL1 patients were subsequently proven as STL1 by genetic testing.

In apparent recognition of the potential risks of more extensive cryopexy, Cambridge did not extend prophylaxis posteriorly and specifically stated that their treatment was not expected to prevent tears posterior to the ora serrata. In contrast, specifically to prevent tears posterior to the ora, we have long practiced a (standard) form of prophylactic

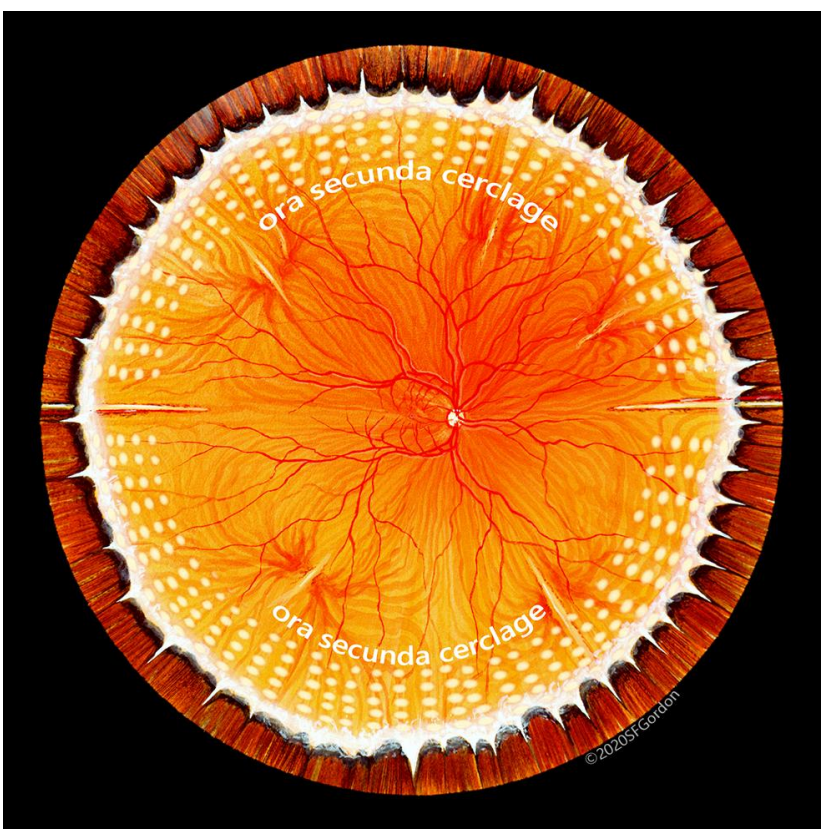

Figure I Illustration of OSC. Laser burns of moderate intensity are placed in a grid pattern (one to two spot widths separation) extending from the ora serrata approximately $4 \mathrm{~mm}$ posteriorly, in effect producing a "second ora" posterior to the vitreous base. Used with permission of artist Stephen Gordon, copyright 2020.

encircling laser retinopexy that has reliably prevented RRD in high-risk, non-syndromic eyes. It emphasizes grid treatment of the at-risk peripheral retina extending from the ora serrata halfway to the vortex vein ampullae. In effect, this creates a "second ora" behind the posterior vitreous base which ends $3 \mathrm{~mm}$ posterior to the ora (Ora Secunda Cerclage, OSC, Figure 1). ${ }^{12,13}$

In our experience, however, the standard OSC prophylaxis of Figure 1 has failed to reliably prevent RRD in Stickler syndrome. Similarly, Alsharani et al recently found no evidence of efficacy in standard (anterior) encircling laser prophylaxis, as $36 \%$ of 70 Stickler eyes presenting with RRD had detached despite such prophylaxis. ${ }^{14}$

However, OSC modified for SS (OSC/SS), to encompass the ora serrata and anterior vitreous base (as per Cambridge) and to extend more posteriorly, has been a successful SS prophylaxis in our experience of the last 8 years. We here describe the OSC/SS technique, illustrated by a single-family series, that may have the potential to become more widely adopted than the Cambridge cryopexy encirclement, based on increased provider familiarity with laser retinopexy.

\section{Patients and Methods}

This retrospective case series study was determined to be exempt from IRB approval by the Western Institutional 
Review Board and was conducted in accordance with the tenets of the Declaration of Helsinki for research involving human subjects. Patients provided written informed consent for their case details to be published.

A 53-year-old man and his 22-year-old son presented with an extensive family history of RRD occurring at an early age. Both the father and son had themselves already suffered RRD (and redetachment) in one eye at age 50 and 18 , respectively, that had been stably reattached after multiple procedures, albeit with severe vision loss. They were in search of therapy to prevent RRD in their fellow eyes.

The man and his son stated that three local retinal specialists had recommended no preventive treatment for their fellow eyes. A 26-year-old daughter and a 28-yearold son were said to be nearsighted, and both had been treated with "spot laser" in each eye for retinal thinning. Another, youngest son was said to have normal eyes without myopia. The father had four siblings, two of whom had suffered retinal detachment at age 17 and at age 31, while two other siblings did not have the Stickler phenotype. His mother had lattice degeneration, early cataract, and glaucoma. His maternal grandfather suffered bilateral retinal detachment by age 47 and was legally blind. The father himself had cataract extraction at age 40 , before suffering retinal detachment in one eye at age 50 .

After each losing substantial vision in one eye to RRD, the father and son had traveled together to an eye institute renowned for its expertise in treatment of retinal disease seeking an additional opinion regarding possible preventive treatment for their fellow, normally sighted eyes. They related that they were again advised to have no laser prophylaxis, but to return promptly for treatment if and when they developed RRD in the fellow eye. They had come to our clinic at the suggestion of a fifth retina specialist.

\section{Examination}

In addition to first eye RRD, features of the Stickler ocular phenotype present in the fellow eyes of the two men included high myopia from birth, optically clear central vitreous, cortical vitreous gel opacities, and lattice retinal degeneration. The father was bilaterally pseudophakic resulting from early-onset cataracts. The father and son had pseudophakic visual acuities of $8 / 200$ and 20/125 in their post RRD eyes, respectively, with visual fields reduced to an average of 27 degrees in each meridian (Figure 2A and B, Figure 3A and B).

A full discussion ensued using diagrams and illustrations of vitreous traction, retinal tears, lattice degeneration, and RRD. A specific form of encircling laser treatment of the peripheral retina in Stickler syndrome (OSC/SS) was described as a possible preventive treatment for their fellow eyes. Included in the discussion were the rationale and the risks of treatment, and the fact that there existed no

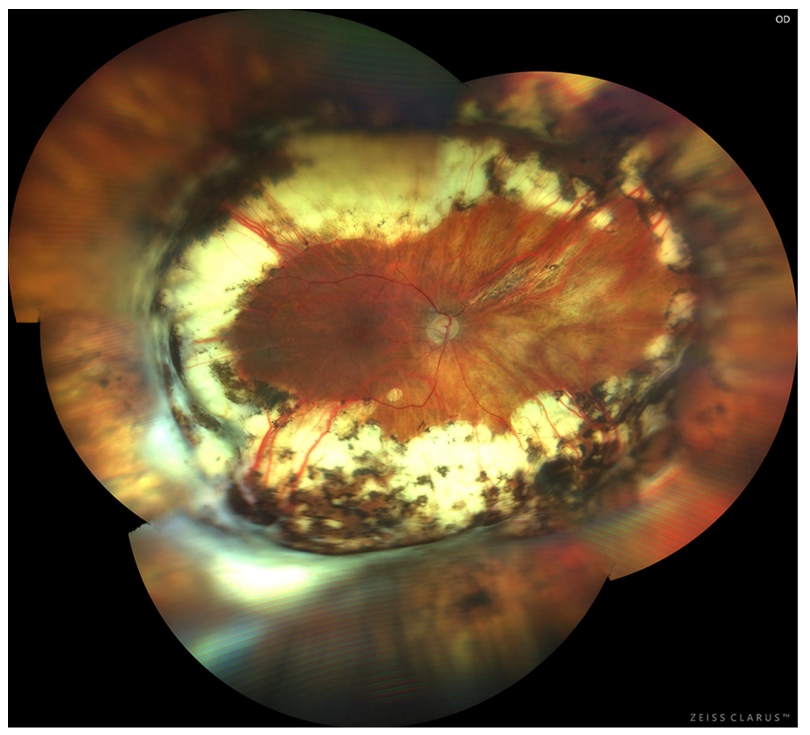

A

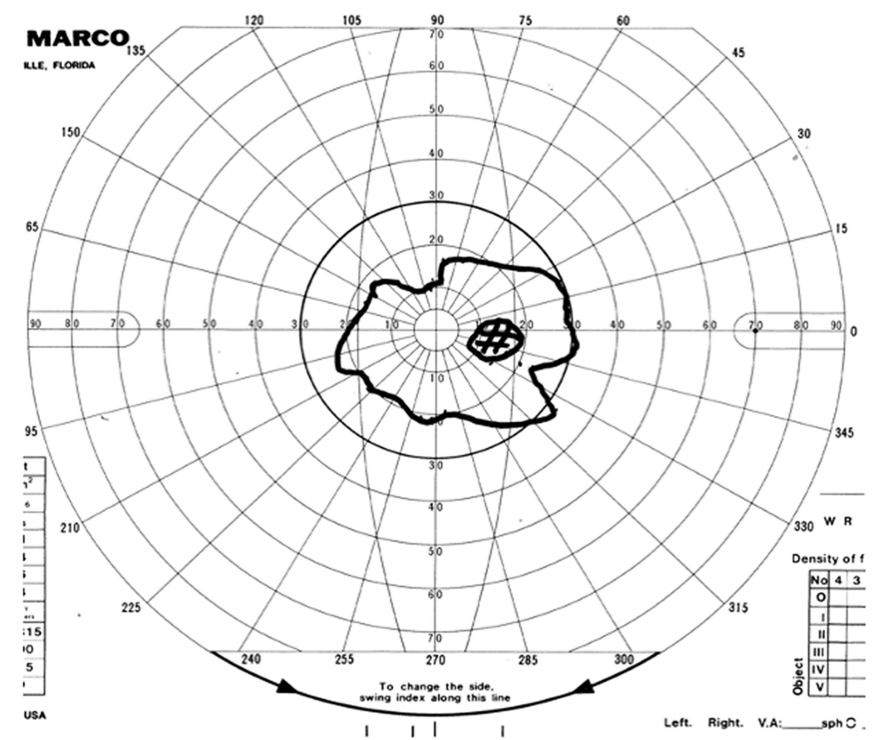

B

Figure 2 (A) Final appearance of the father's right eye fundus after repair of recurrent RRD/PVR in multiple procedures. Postoperative visual acuity is 8/200. Multiple causative tears with aberrant vitreous traction were noted at RRD repair. (B) Visual field of the right eye postoperatively. 


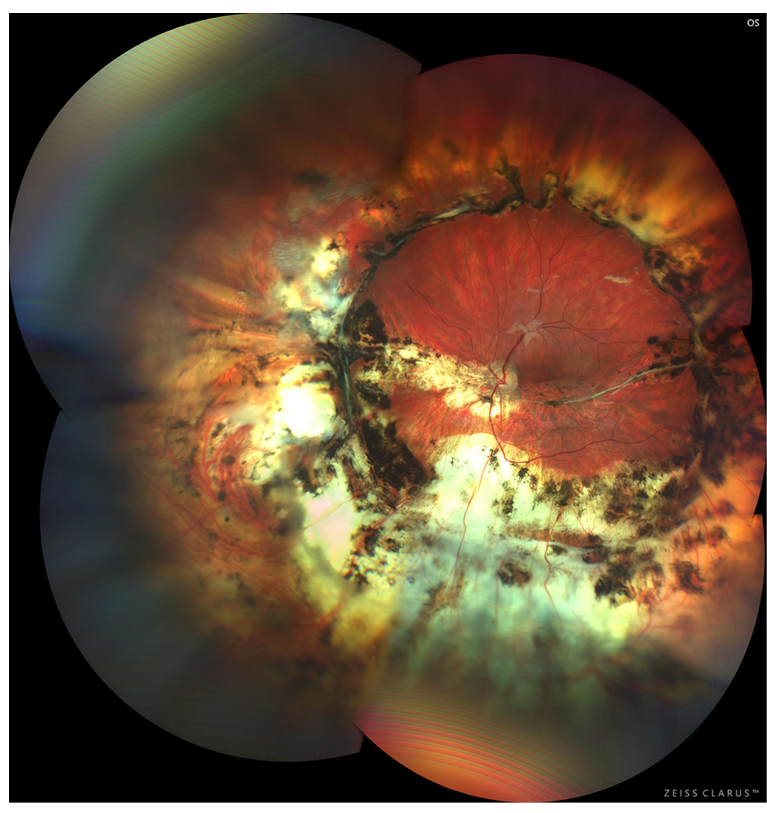

A

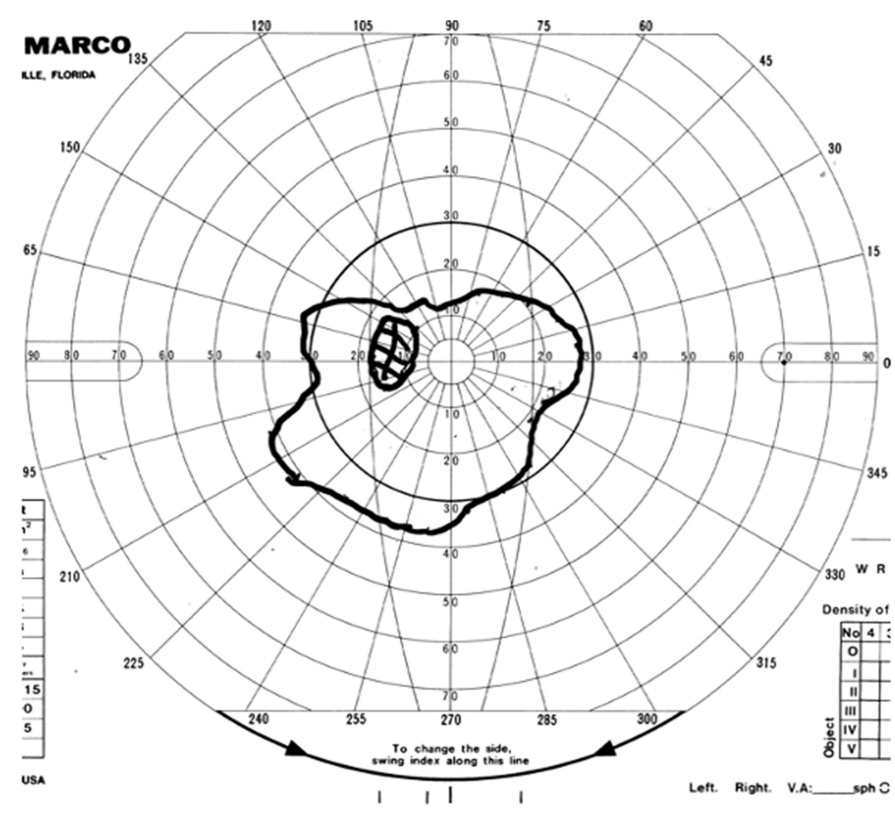

B

Figure 3 (A) Final appearance of the son's left eye after repair of recurrent RRD/PVR. Postoperative visual acuity is 20/I25. Initial RRD was from multiple small defects with lattice. (B) Visual field of the left eye postoperatively.

prospective clinical trials of this or any other prophylactic procedure. ${ }^{15}$

The alternatives described were focal retinopexy limited to visible lattice; ${ }^{15}$ encircling scleral buckling (more invasive, and itself not proven); ${ }^{16}$ encircling cryopexy (performed primarily at Cambridge); ${ }^{9,17}$ or continued observation, accepting the natural course of Stickler syndrome. ${ }^{9}$

\section{Treatment}

Both the father and the presenting son chose OSC/SS prophylaxis for their fellow eye, gave written informed consent prior to each treatment, and were treated with laser delivery via the indirect ophthalmoscope (IDO) under laryngeal mask general anesthesia (LMA) without complications (Figure 4A and B).

The 26-year-old daughter was subsequently examined, with similar findings consistent with Stickler phenotype, including myopia of 20 diopters, 3.75 diopters of astigmatism, an optically clear vitreous with "stringy" opacities in the cortical vitreous gel, and lattice retinal degeneration. She had modest focal laser prophylaxis to lattice OU. Her corrected visual acuity was 20/25 right eye and 20/30 left eye. She was offered OSC/SS in each eye for her consideration.
While contemplating possible treatment, 6 weeks later she suffered RRD from a six clock hour GRT in the left eye that was reattached in her hometown using silicone oil. Even after silicone oil removal and cataract extraction, maculopathy limited her final corrected visual acuity to $20 / 125$ in the left eye and visual field was reduced to an average of 32 degrees in each meridian. She then chose OSC/SS prophylaxis in her fellow right eye and was treated under LMA in two sessions after written informed consent.

A 28-year-old son was found to have eight diopters of myopia, early onset cataracts, abnormally clear central vitreous, cortical vitreous opacities, and lattice retinal degeneration. He had minimal laser treatment to areas of thin retina in both eyes. He chose OSC/SS prophylaxis, gave written informed consent, and was treated OU under LMA over a period of 4 years on a schedule of his choice. He then had successful cataract extraction in his right eye. After subsequent symptomatic posterior vitreous detachment in the left eye 2 years after final OSC/SS treatment, he gave written informed consent and underwent left eye combined cataract extraction and 27-gauge vitrectomy with removal of symptomatic vitreous veil opacities.

The father, his three affected children, and a 4-year-old grandson were each tested for mutations in the genes known to be involved in Stickler syndrome. No family 

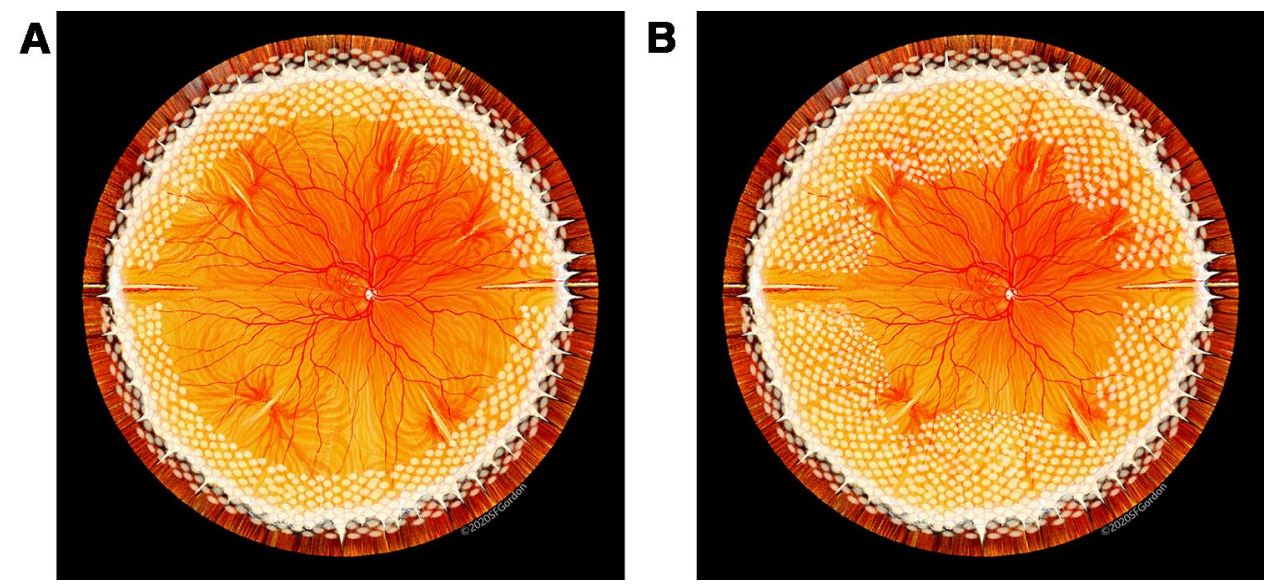

Figure 4 (A) Step I (essential) of OSC/SS. Laser burns of moderately high intensity are placed in a tight grid pattern (one spot width separation) from 2 mm onto the pars plana to the ora serrata, and approximately $4 \mathrm{~mm}$ posteriorly, halfway to the vortex vein ampullae, achieving protection against GRT and anterior defects. Artist Stephen Gordon. (B) Step 2 (optional) of OSC/SS. Three months after initial treatment, the laser grid is extended posteriorly to and between the vortex vein ampullae, achieving maximum protection against both GRT and posterior defects throughout the peripheral retina. Used with permission of artist Stephen Gordon, copyright 2020.

member was noted to have skeletal abnormalities. A hearing test was performed on the father.

\section{Results}

None of the five eyes in the four family members treated by OSC/SS prophylaxis experienced either a retinal tear or an RRD with an average follow-up of 8.7 years (Video S1). Each of these five eyes maintained preoperative corrected visual acuity of $20 / 20$ to $20 / 30$, with an asymptomatic reduction of visual field to an average of 50 degrees in each meridian post-prophylaxis. The three fellow eyes treated with OSC/SS have now gone 12, 11, and 8 years after first eye RRD without suffering a rhegmatogenous event. The daughter's right eye fundus image and visual field, typical of the OSC/SS treated eyes, are shown in Figure 5A and B, respectively.

At vitrectomy performed in the left eye, the eldest son was noted to have both moderate beading opacities characteristic of STL2 and extensive membranous vitreous veils (Video S2), distinct however from the anterior membranous

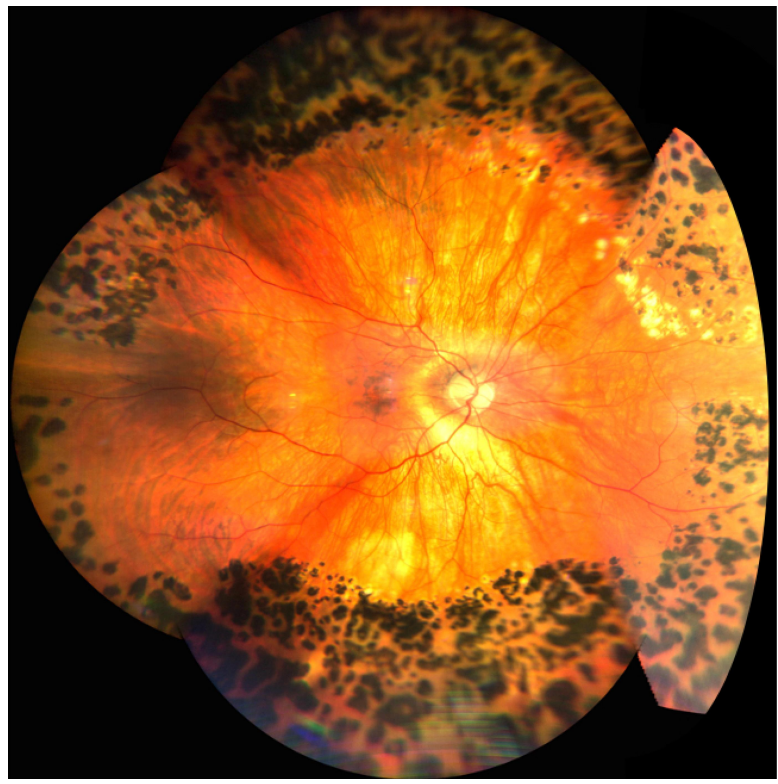

A

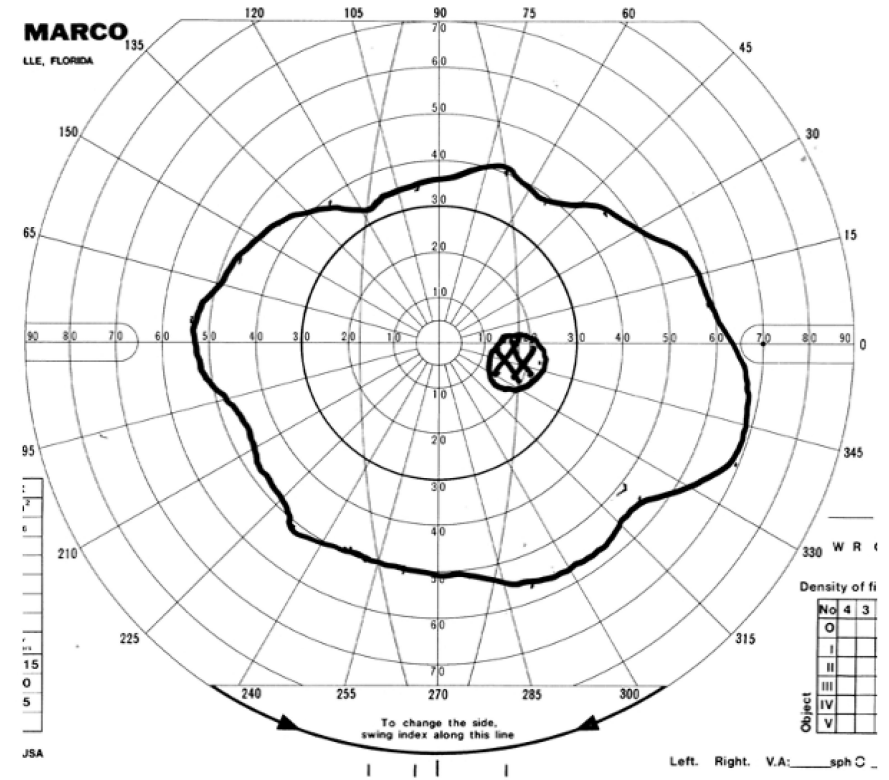

B

Figure 5 (A) Fundus image of the daughter's right eye after completed OSC/SS laser prophylaxis. Visual acuity is $20 / 30$ (as preoperatively) corrected with $-20.25+3.75 \times$ 09I. (B) Visual field of the right eye post OSC/SS. 
opacity characteristic of STL1 eyes. ${ }^{3,18}$ He ultimately achieved 20/25 uncorrected visual acuity in the right eye and 20/30 uncorrected near visual acuity in the left eye.

Of the five OSC/SS-treated eyes, one developed pupillary mydriasis that was moderately symptomatic and persisted for 6 months, but ultimately resolved. No eye developed epimacular proliferation, vitreous traction exacerbated by treatment, or any other complication.

Each affected family member was found to have an identical "variant of unknown significance" mutation in the gene that is associated with STL2 (Col11A1), less common than STL1, but having similar RRD propensity. ${ }^{19}$ The father had moderate sensorineural hearing loss. The father and four of his five affected siblings/children suffered RRD (5/6, $83 \%$ ). The one affected child not developing RRD had received OSC/SS prophylaxis OU, with 7-year follow-up in the right eye and 3-year follow-up in the left eye.

\section{Discussion}

\section{The Stickler Problem}

The fundamental problem in Stickler eyes is that the vitreous cavity is only partially filled with gel vitreous from birth. ${ }^{5}$ The central vitreous does not "liquify" - it never forms. And aqueous fluid is ready to breach any retinal hole or tear that develops. Cortical vitreous movements and contraction (Video S3) can give rise to tractional retinal tears, completely independently of posterior vitreous detachment, at an age when normal eyes with a completely gel-filled vitreous cavity are impervious to such an occurrence. ${ }^{5}$ In fact, less than half of Stickler RRDs have posterior vitreous detachment (Video S3). ${ }^{14}$ Moreover, the vitreous gel that is present adheres to the retina in a grossly anomalous and unpredictable fashion. Finally, lattice and perivascular retinal degeneration and strong foci of vitreous adherence can extend quite posteriorly (Figure 6). ${ }^{4,18}$

As a consequence of these vitreoretinal abnormalities, and in the absence of prophylaxis, most STL1 (and probably STL2, as in this family) ${ }^{19}$ Stickler patients experience RRD, half by age 20 , with an $80 \%$ probability of fellow eye RRD within a median of 4 years in the largest observational report to date. ${ }^{9}$ Two of three affected children in the current family had first eye RRD by age 26 , and one of three detachments in this family was from GRT. Yet three fellow eyes treated by OSC/SS laser retinopexy have gone 12 years, 11 years, and 8 years after first eye detachment without developing RRD.

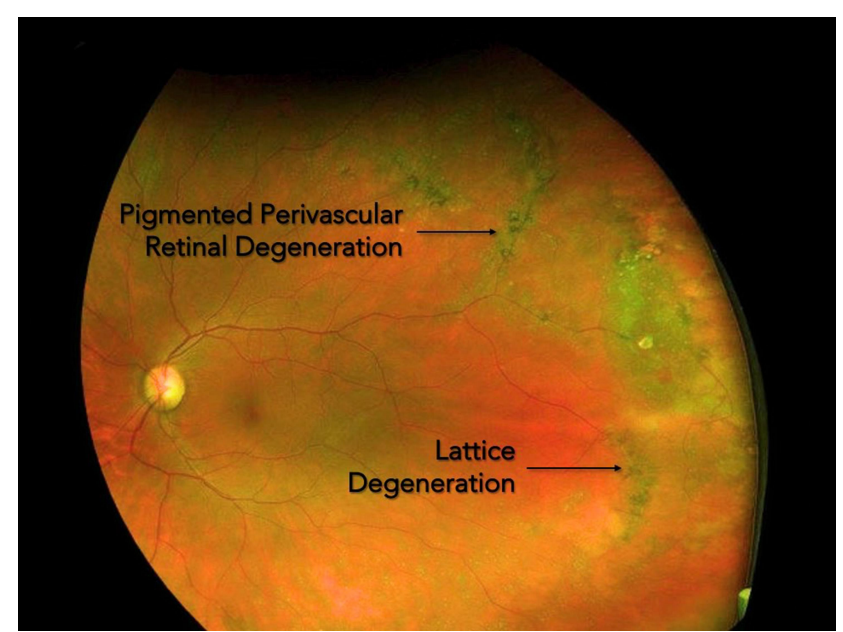

Figure 6 Fundus image of pigmented perivascular retinal degeneration extending radially and posteriorly, as seen in some Stickler syndrome pedigrees.

Prevention of RRD by adoption of the Cambridge cryopexy protocol has been hindered by the fact that cryopexy is now used very sparingly by most retina specialists, who regard laser retinopexy as superior, and who have never in their careers performed encircling cryopexy. ${ }^{18,21}$ Focal cryopexy and laser retinopexy are each successful prophylaxis in the hands of surgeons adept at them. However, the majority of current practitioners are insufficiently skilled and reluctant to perform encirclement with cryopexy, when the laser retinopexy they expertly perform almost daily is available as a reasonable encircling prophylaxis alternative. ${ }^{18}$

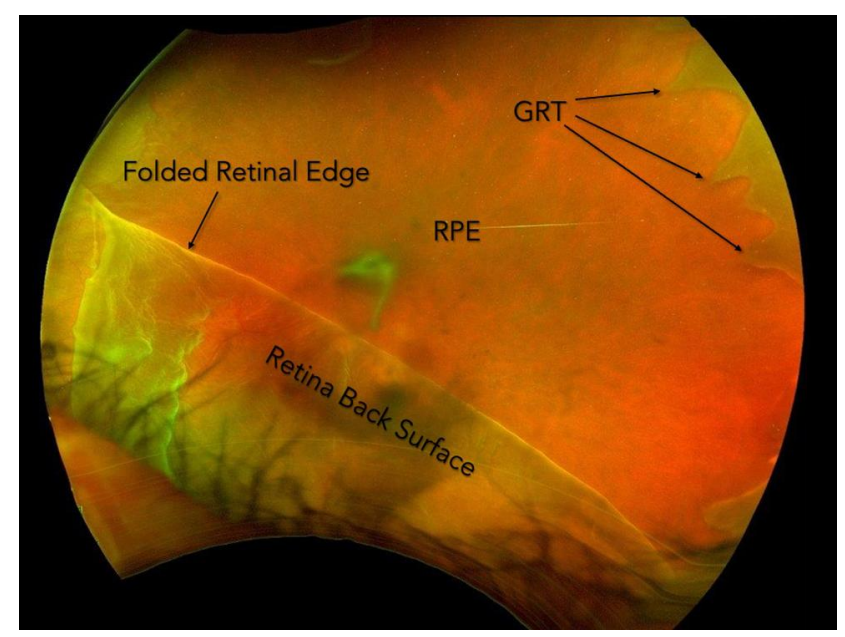

Figure 7 Fundus image of a giant retinal tear (GRT) extending from 9 to 5 o'clock in the left eye. GRT is most commonly seen in childhood retinal detachments of Stickler syndrome patients. 


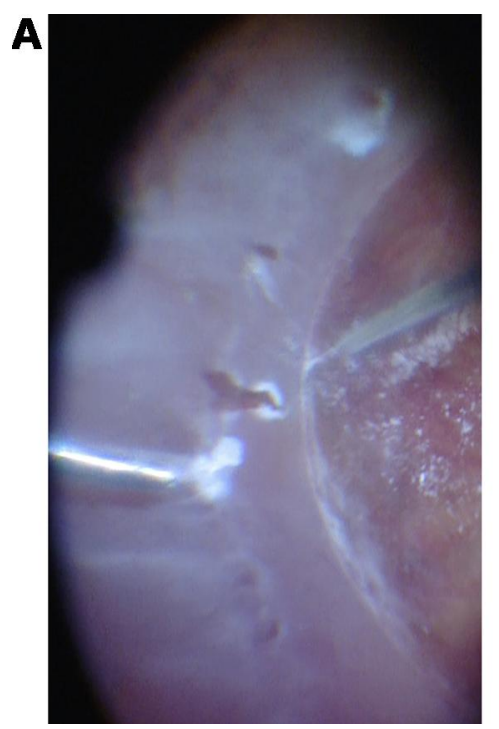

B

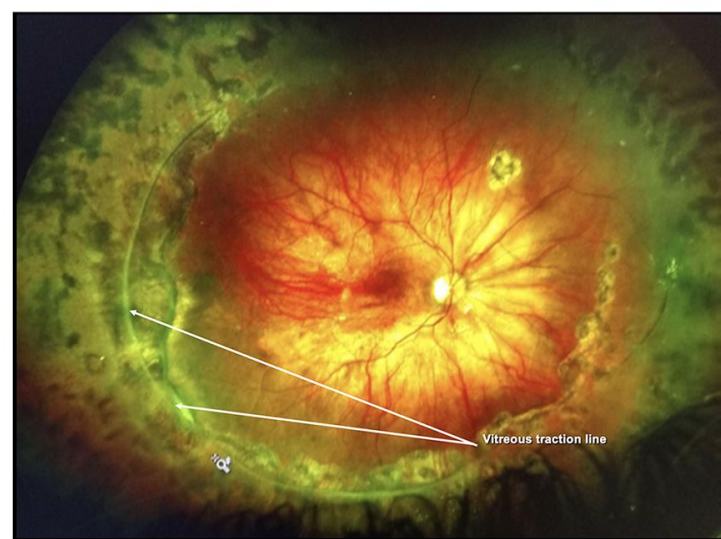

Figure 8 (A) Intraoperative image of seven post equatorial retinal tears extending from 8 to 10 o'clock temporally along a prominent circumferential line of vitreous traction, causing total RRD in the right eye of a 14-year-old female with STLI, congenital extreme myopia (26 diopters), and abnormal vitreous (Video S3). These tears occurred well posterior to standard OSC prophylaxis performed 4 months previously. (B) Postoperative image of the same retina reattached under silicone oil, with corrected visual acuity in the eye of 20/30. Note the prominent line of vitreous traction from 5 to II o'clock that could not be safely removed during the initial repair despite retinal stabilization with perfluorocarbon liquid. Laser retinopexy extends quite posteriorly to encompass all tears and the traction line that was further reduced upon silicone oil removal.

\section{OSC/SS Technique}

In our experience, Stickler RRD emanating from either GRT (Figure 7; one of three detachments in this family) or from multiple posterior tears (Figure 8A and B; Video S3; two of three detachments in this family) can be reliably prevented by IDO delivery of a dense grid of laser retinopexy starting $2 \mathrm{~mm}$ anterior to the ora, extending to and between the vortex vein ampullae posteriorly. OSC/SS employs slightly higher power and a tighter grid pattern (one spot width separation) than in standard OSC, as illustrated in Figure 4A and B.

This two-step prophylaxis emulates the successful Cambridge GRT prophylaxis in Step 1. But in Step 2, it also attempts to prevent the $9 \%$ Cambridge failure rate over 5.6 years of follow-up, due predominately to new posterior tears. ${ }^{9}$ Step 2 further safeguards treated eyes for a life expectancy averaging decades beyond the mean age at which Cambridge cryopexy was performed (14.5 years bilateral prophylaxis; 22.9 years unilateral prophylaxis).

Treatment of one or both eyes of young, inherently high-risk Stickler patients is best performed in the operating room under laryngeal mask general anesthesia (LMA). In an initial treatment session (Step 1), laser grid treatment is placed from $2 \mathrm{~mm}$ anterior to the ora serrata (reaching the anterior vitreous base) to $4 \mathrm{~mm}$ posterior to the ora (posterior to the normal vitreous base at $3 \mathrm{~mm}$ from the ora), about halfway to the vortex vein ampullae, taking care to spare the long posterior ciliary nerves at the three and nine o'clock meridians (Figure 4A). Step 1 is the essential step in achieving security for central vision, with additional steps as described below to be elected by each patient after further counseling, so as to achieve the patient's maximum desired prophylaxis.

After a normal recovery from Step 1 laser retinopexy, without anterior segment sequelae, ${ }^{22}$ (or as a supplement to Cambridge cryopexy encirclement) a laser treatment session 3 months later (Step 2) fills in skip areas and extends further posteriorly to and between carefully localized vortex vein ampullae. No treatment is applied directly overlying visible vortex vein ampullae or their posterior choroidal drainage vessels. Once sufficient experience accumulates to assure safety and effectiveness, it may be possible to offer a trial of OSC/SS in a single treatment.

Total OSC/SS treatment is approximately 2000 to 2400 spots (Figure 4B). If a shallow fornix limits the scleral depression needed to achieve adequate posterior coverage, an access conjunctival incision can be used. Alternatively, the posterior extent of treatment can be incrementally adjusted using a wide-angle contact lens and a slit lamp in the clinic during a final session with topical anesthesia.

Beyond accurate grid laser placement, good control of laser power is critically important. The delicate 
neurosensory retina can develop necrotic defects when the chorioretinal complex is treated with excessive power density or overlapping burns, weakening the retina instead of strengthening it as intended. The definition of "burn" is "to alter or destroy by the action of heat." 23 The distinction between altering to produce a strengthening adhesion, and destroying by causing retinal necrosis can be minimal, requiring vigilance in monitoring grid placement, power density and the resultant retinal "whitening."

\section{Posterior Prophylaxis Uncertainty}

Optimal prophylaxis in SS must extend more posteriorly than in non-syndromic eyes because both retinal degeneration and anomalous vitreous traction commonly extend more posteriorly. ${ }^{20}$ But any encircling laser retinopexy reduces the peripheral visual field while increasing central vision security, and this trade-off increases in OSC/SS prophylaxis as the retinopexy border is extended more posteriorly. Potentially greater loss of visual field understandably gives rise to controversy. Yet even after Step 2 of OSC/SS retinopexy, the loss of peripheral field was asymptomatic in all four treated members of this family and rated as a $0 \%$ disability by Estermann scoring. ${ }^{24}$

In a 2019 review, Coussa et al noted that encircling prophylaxis is commonly performed in fellow eyes when an initial Stickler RRD is repaired, but that the optimal posterior extent of treatment has not been established. ${ }^{25}$ While posterior tears causing retinal detachment are relatively rare in non-syndromic eyes, in a series of Stickler detachments, Billington et al noted multiple small tears with "many post equatorial and at different distances from the ora serrata." 26

In 1968, just 3 years after Gunnar Stickler's first report, Hagler reported on 33 patients with familial radial perivascular retinal degeneration (Figure 6) and ocular features that have subsequently been shown to characterize SS, including 22 retinal detachments. ${ }^{20}$ None were caused by GRT, but three related family members suffered GRT detachments. Hagler reported a $36 \%$ failure rate in detachment repair due to multiple posterior tears and thus referred to these as "malignant" detachments. In 2002, Parma et al showed that this same pedigree was indeed genetically STL1. RRD occurred in $65 \%$ of 100 patients, with half bilaterally, and $70 \%$ of detachments occurred by age $18 .^{4}$

In a total of four literature reports specifically analyzing Stickler RRD, approximately 82 of 158 eyes $(52 \%)$ had multiple small tears, while 43 eyes $(27 \%)$ had giant retinal tears. $^{10,14,26,27}$ Based on these reports and an estimated lifetime RRD risk in SS patients of over $65 \%,{ }^{4,17}$ one would expect at least a $30 \%$ remaining risk of RRD from posterior tears in SS patients even after GRT prophylaxis. Thus, posterior prophylaxis via Step 2 OSC/SS laser retinopexy can reasonably be offered to all STL1 and STL2 patients. The Cambridge operative experience with over 250 Stickler detachments might considerably enhance our knowledge of causative tear locations, further defining the posterior prophylaxis (beyond GRT prophylaxis at the ora) needed to optimally secure central vision for a lifetime. ${ }^{9}$

The extent of laser retinopexy preventive treatment and placement of the posterior border of prophylaxis in Stickler eyes are ultimately decisions to be made in each eye based on each physician's judgment and each patient's preference (Supplemental Report). For the three family members who lost vision due to RRD in one eye, choosing maximum retinopexy prophylaxis by both Step 1 and Step $2 \mathrm{OSC} / \mathrm{SS}$ was an easier decision. Similarly, other Stickler patients with one eye detachment or with a multigenerational family history of detachment would likely be so inclined.

\section{Vitrectomy as Prophylaxis}

It is indisputable that STL1 and STL2 patients are at extreme risk for RRD, and that tractional tears account for the preponderance of these detachments. ${ }^{9,10,14,17-19,25-27}$ Consequently, 27-gauge vitrectomy to prevent vitreous traction (carrying less than a $1 \%$ risk in our experience, when performed with specific precautions) is now a reasonable, final preventive option (Step 3) to be considered in selected eyes after completion of OSC/SS retinopexy. Substantial advances in ultra-high-speed vitrectomy probes, low-end suction control, Kenalog vitreous staining, and wide-field microscopes have finally made Step 3 a reasonably safe alternative to complete reliance on prophylactic retinopexy. This final step helps SS eyes avoid a lifetime of vitreous tractional events that are more common but less predictable (as to location) in Stickler eyes than in non-syndromic eyes. As an example, the seven tears seen in the Stickler RRD of Video S3 were so posterior that they likely would not have been prevented even by the maximum OSC/SS retinopexy prophylaxis here described.

Alsharani et al found that vitreous veils were present at the site of tears in 70 consecutive Stickler detachments. ${ }^{14}$ Such opacities (Figure 9) may help guide vitreous removal as a final prophylactic measure in some Stickler eyes, as in the vitrectomy performed in the older son of this family 


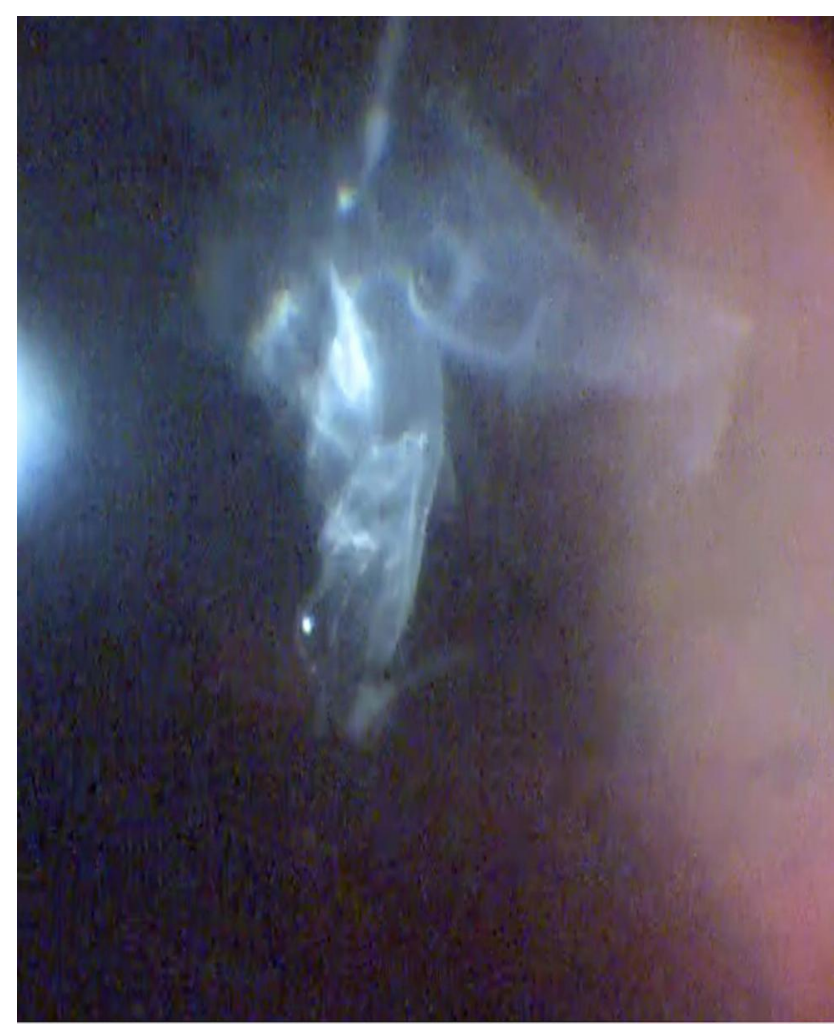

Figure 9 Intraoperative image of vitreous veils in the left eye of the elder son, an STL2 patient (Video S2).

(Video S2). Moreover, Stickler patients who present with symptomatic vitreous opacities may be at especially high risk of detachment. In fact, all Stickler patients and their parents should be well educated to promptly report premonitory signs of vitreous traction for professional assessment.

\section{Conclusion}

A recent comprehensive analysis of Stickler syndrome RRD prophylaxis by the National Institute for Health Research of the United Kingdom concluded that a prospective, randomized trial of prophylaxis in this rare disease was impractical and instead recommended a nonrandomized, prospective, cohort comparison study of ongoing individual efforts. ${ }^{28}$ We hope that dissemination of the OSC/SS technique will be another significant step, building on the pioneering work of the Cambridge group, towards the goal of developing an effective, statistically validated prophylaxis for RRD in Stickler syndrome.

$\mathrm{OSC} / \mathrm{SS}$ is a noninvasive treatment whose sole purpose is risk reduction, and the risk of treatment itself has been minimal when applied appropriately. ${ }^{12,13,18}$ However, each physician should remain mindful that such treatment is tantamount to a permanent laser signature and to always "laser" as much as necessary but as little as possible, with great care befitting the permanent changes inevitably attending such retinopexy.

Encircling prophylaxis is a reasonable option for Stickler patients to consider. And yet after undergoing five retinal detachment repairs with final profound visual loss in three family members, and after being seen by four retinal specialists over a period of years, none of the four affected family members had learned of either the widely used encircling laser prophylaxis option, ${ }^{12,13,29-36}$ or the Cambridge encircling cryopexy prophylaxis for their fellow eyes. Even in the absence of level-one evidence of effectiveness, ${ }^{15}$ we must provide Stickler patients with all the information we have, enabling them to make their own fully informed prophylaxis choice. For in the absence of effective RRD prevention they spend each day "under the sword of Damocles," with the eminent and ever-present danger of sudden blindness. ${ }^{37}$

\section{Data Sharing Statement}

Additional material related to this article can be found at www.helenkellerfoundation.org.

\section{Ethics and Consent Statement}

This retrospective case series study was determined to be exempt from IRB approval by the Western Institutional Review Board and was conducted in accordance with the tenets of the Declaration of Helsinki for research involving human subjects. Patients provided written informed consent for their case details to be published.

\section{Acknowledgments}

As in many prior articles, the authors are grateful for excellent research, clinical, and clerical support by Christina Sullivan, Jessica Haynes, Dewayne Conn, and Laura Beckwith. C. Diane Scharper has again provided expert editing services. The authors also appreciate the supportive environment provided by the staff and physicians of Retina Specialists of Alabama and the UAB Callahan Eye Hospital.

\section{Author Contributions}

Dr. Robert Morris initally conceptualized OSC/SS, performed all treatments, and wrote the first draft. All additional authors made substantial contributions to conception and design, acquisition of data, or analysis and 
interpretation of data; took part in drafting the article or revising it critically for important intellectual content; agreed to submit to the current journal; gave final approval of the version to be published; and agree to be accountable for all aspects of the work.

\section{Funding}

Partial funding was provided by the Helen Keller Foundation for Research and Education, through grants by the Hanna Charitable Trust and the Kent Companies, and by Retina Specialists of Alabama LLC.

\section{Disclosure}

The authors report no conflicts of interest in this work financial or otherwise.

\section{References}

1. Robin NH, Moran RT, Warman M, et al. Stickler syndrome. In: Pagon RA, Adam MP, Bird TD, editors. GeneReviews. Seattle: University of Washington, Seattle; 2017.

2. Richards AJ, McNinch A, Martin H, et al. Stickler syndrome and the vitreous phenotype: mutations in COL2A1 and COL11A1 [report online]. Hum Mutat. 2010;31(6):E1461-71. doi:10.1002/humu.21257

3. Snead MP, McNinch AM, Poulson AV, et al. Stickler syndrome, ocular-only variants and a key diagnostic role for the ophthalmologist. Eye (Lond). 2011;25(11):1389-1400. doi:10.1038/ eye.2011.201

4. Parma ES, Körkkö J, Hagler WS, Ala-Kokko L. Radial perivascular retinal degeneration: a key to the clinical diagnosis of an ocular variant of stickler syndrome with minimal or no systemic manifestations. Am J Ophthalmol. 2002;134(5):728-734. doi:10.1016/S0002-9394(02)01646-X

5. Watanabe Y, Ueda M, Adachi-Usami E. Retinal detachment in identical twins with stickler syndrome type 1. Br J Ophthalmol. 1996;80 (11):976-981. doi:10.1136/bjo.80.11.976

6. Matsushita I, Nagata T, Hayashi T, et al. Foveal hypoplasia in patients with stickler syndrome. Ophthalmology. 2017;124 (6):896-902. doi:10.1016/j.ophtha.2017.01.046

7. Snead MP, Yates JR. Clinical and molecular genetics of stickler syndrome. J Med Genet. 1999;36:353-359.

8. Stickler GB, Belau PG, Farrell FJ, et al. Hereditary progressive arthro-ophthalmopathy. Mayo Clin Proc. 1965;40:433-455.

9. Fincham GS, Pasea L, Carroll C, et al. Prevention of retinal detachment in stickler syndrome. the cambridge prophylactic cryotherapy protocol. Ophthalmology. 2014;121(8):1588-1597. doi:10.1016/j. ophtha.2014.02.022

10. Reddy DN, Yonekawa Y, Thomas BJ, et al. Long-term surgical outcomes of retinal detachment in patients with stickler syndrome. Clin Ophthalmol. 2016;10:1531-4.

11. Boysen KB, La Cour M, Kessel L. Ocular complications and prophylactic strategies in stickler syndrome: a systematic literature review. Ophthalmic Genet. 2020;41(3):223-234. doi:10.1080/ 13816810.2020.1747092

12. Morris RE, Shere JL, Witherspoon CD, et al. Intraoperative retinal detachment prophylaxis in vitrectomy for retained cataract fragments. J Cataract Refract Surg. 2009;35(3):491-495. doi:10.1016/j.jcrs.2008.11.037

13. Morris R, Witherspoon CD, Kuhn F, Sapp M. Retinal detachment laser prophylaxis. Retin Today. 2008;63-66.
14. Alshahrani ST, Ghazi NG, Al-Rashaed S. Rhegmatogenous retinal detachments associated to stickler syndrome in a tertiary eye care center in Saudi Arabia. Clin Ophthalmol. 2015;10:1-6.

15. American Academy of Ophthalmology Preferred Practice Pattern. Preferred practice guidelines. posterior vitreous detachment, retinal breaks, and lattice degeneration. San Francisco, CA. American Academy of Ophthalmology; 2019. Available from: www.aao.org/ ppp. Accessed November 18, 2020.

16. Monin C. Prevention of non-traumatic retinal detachment by surgical cerclage. Apropos of 20 cases. J Fr Ophtalmol. 1993;16(4):247-253.

17. Ang A, Poulson AV, Goodburn SF, et al. Retinal detachment and prophylaxis in type I stickler syndrome. Ophthalmology. 2008;115 (1):164-168. doi:10.1016/j.ophtha.2007.03.059

18. Shapiro MH, Blair MP, Solinski MA, et al. The importance of early diagnosis of stickler syndrome: finding opportunities for preventing blindness. Taiwan J Ophthalmol. 2018;8(4):189-195. doi:10.4103/ tjo.tjo_97_18

19. Poulson AV, Hooymans JMM, Richards AJ, et al. Clinical features of type 2 stickler syndrome. J Med Genet. 2004:41e107. Available from: http:// www.jmedgenet.com/cgi/content/full/41/8/e107. Accessed December 9, 2020.

20. Hagler WS, Crosswell HH Jr. Radial perivascular chorioretinal degeneration and retinal detachment. Trans Am Acad Ophthalmol Otolaryngol. 1968;72(2):203-216.

21. Campochiaro PA, Gaskin HC, Vinores SA. Retinal cryopexy stimulates traction retinal detachment formation in the presence of an ocular wound. Arch Ophthalmol. 1987;105(11):1567-1570. doi:10.1001/archopht.1987.01060110113043

22. Bouheraoua N, Hrarat L, Parsa CF, et al. Decreased corneal sensation and subbasal nerve density, and thinned corneal epithelium as a result of 360-degree laser retinopexy. Ophthalmology. 2015;122 (10):2095-2102. doi:10.1016/j.ophtha.2015.06.010

23. Editors. Definition: "burn", Merriam-Webster Dictionary. Available from: https://www.merriam-webster.com/dictionary/burn. Accessed September 24, 2020.

24. Estermann B. Grid for scoring visual fields. II. perimetry. Arch Ophthalmol. 1968;79(4):400-406. doi:10.1001/archopht.1968. 03850040402007

25. Coussa RG, Sears J, Traboulsi EI. Stickler syndrome: exploring prophylaxis for retinal detachment. Curr Opin Ophthalmol. 2019;30 (5):306-313. doi:10.1097/ICU.0000000000000599

26. Billington BM, Leaver BK, McLeod D. Management of retinal detachment in the wagner-stickler syndrome. Trans Ophthalmol Soc UK. 1985;104(8):875-879.

27. Abeysiri P, Bunce C, da Cruz L. Outcomes of surgery for retinal detachment in patients with stickler syndrome: a comparison of two sequential 20-year cohorts. Graefes Arch Clin Exp Ophthalmol. 2007;245(11):1633-1638. doi:10.1007/s00417-007-0609-2

28. Carroll C, Papaioannou D, Rees A, Kaltenthaler E. The clinical effectiveness and safety of prophylactic retinal interventions to reduce the risk of retinal detachment and subsequent vision loss in adults and children with stickler syndrome: a systematic review. Health Technol Assess. 2011;15(16):Iii-xiv, 1-62. doi:10.3310/ hta 15160

29. Haut J, Faure JF, Larricart P, Monin C, Le Mer Y. Treatment by internal tamponade of retinal detachment with macular hole. $J \mathrm{Fr}$ Ophtalmol. 1987;10(11):707-715.

30. Madelain J. Results of systematic preventive treatment with argon laser after idiopathic retinal detachment.. Bull Soc Ophtalmol Fr. 1990;90(2):141-147.

31. Tufail A, Schwartz SD, Gregor ZJ. Prophylactic argon laser retinopexy prior to removal of silicone oil: a pilot study. Eye. 1997;11 (3):328-330. doi:10.1038/eye.1997.69

32. Ahluwalia HS, Gray RH. Prophylactic argon laser retinopexy prior to removal of silicone oil: a pilot study (editorial). Eye. 1998;12(3):490. doi:10.1038/eye.1998.121 
33. Laidlaw DAH, Karia N, Bunce C, Aylward GW, Gregor ZJ. Is prophylactic 360_ laser retinopexy protective? Risk factors for retinal redetachment after removal of silicone oil. Ophthalmol. 2002;109 (1):153-158. doi:10.1016/S0161-6420(01)00848-X

34. Koh HJ, Cheng L, Kosobucki B, Freeman WR. Prophylactic intraoperative 360_ laser retinopexy for prevention of retinal detachment Retina. 2007;27(6):744-749. doi:10.1097/IAE.0b013e318030ebd7

35. Verhoekx JSN, van Etten PG, Wubbels RJ, et al. Prophylactic laser treatment to decrease the incidence of retinal detachment in fellow eyes of idiopathic giant retinal tears. Retina. 2020;40(6):1094-1097. doi:10.1097/IAE.0000000000002494
36. Dirani A, Antaki F, Rhéaume MA, et al. 360-degree intra-operative laser retinopexy for the prevention of retinal re-detachment in patients treated with primary pars plana vitrectomy. Graefes Arch Clin Exp Ophthalmol. 2020;258(2):249-256. doi:10.1007/s00417019-04534-5

37. Editors. The sword of damocles. Collins Dictionary. Available from: https://www.collinsdictionary.com/us/dictionary/english/sword-ofdamocles. Accessed May 4, 2020.

\section{Publish your work in this journal}

Clinical Ophthalmology is an international, peer-reviewed journal covering all subspecialties within ophthalmology. Key topics include: Optometry; Visual science; Pharmacology and drug therapy in eye diseases; Basic Sciences; Primary and Secondary eye care; Patient Safety and Quality of Care Improvements. This journal is indexed on PubMed

Submit your manuscript here: https://www.dovepress.com/clinical-ophthalmology-journal
Central and CAS, and is the official journal of The Society of Clinical Ophthalmology (SCO). The manuscript management system is completely online and includes a very quick and fair peer-review system, which is all easy to use. Visit http://www.dovepress.com/ testimonials.php to read real quotes from published authors. 